\title{
Quo vadis, Energiewenderecht?
}

\author{
Energierechtliche Zukunftsgestaltung
}

Michael Kalis, IKEM - Institut für Klimaschutz, Energie und Mobilität e.V., Magazinstraße 15-26, 10179 Berlin (michael.kalis@ikem.de)

Lars Dittmar, IKEM - Institut für Klimaschutz, Energie und Mobilität e. V. (lars.dittmar@ikem.de) @10 orcid.org/0000-0003-4949-6273

Die Energiewende betrifft als Transformationsprozess eine Vielzahl von Beteiligten: Unternehmen, Forschung, Verbraucher*innen und den Gesetzgeber. Letzterer kann ein erheblicher Treiber oder ein vehementer Bremser der Energiewende sein. Das Energiewenderecht entscheidet maßgeblich über das Gelingen oder Misslingen der Energiewende und ist damit Teil der energierechtlichen Zukunftsgestaltung. Diese Energiezukunft braucht technologische, ökonomische und regulatorische Innovationen. Angesichts des schwierigen Umgangs mit Innovationen steht der Gesetzgeber vor besonderen Herausforderungen. Zur Bewältigung dieser hat er einen breiten Instrumentenkanon zur Verfügung. Im Fokus stehen derzeit unter anderem sogenannte Experimentierklauseln. Ob das Energiewenderecht hiermit auf die Technologien und Geschäftsmodelle der Zukunft vorbereitet ist, untersuchen die Autoren in diesem Aufsatz.

\section{Quo vadis, energy transition law?}

Shaping the future energy system

As a transformation process, the energy transition involves a variety of actors: enterprises, science, consumers, and the legislator. The latter can be a major driver or inhibitor of the energy transition. The energy transition law is decisive for the success or failure of the energy transition and plays a part in shaping the future energy system. This energy future requires technological, economic, and regulatory innovations. Given the complexity of innovations, the legislator faces special challenges. It has a wide range of legal instruments at its disposal to deal with these challenges. A current focus is on so-called experimentation clauses. In this article, the authors examine whether these instruments are sufficient to prepare the energy transition law for the technologies and business models of the future.

Keywords: innovation, deregulation, experimental rule, carbon pricing

\section{Entwicklungen des Energiewenderechts}

Das erste Energiewirtschaftsgesetz (EnWG) aus dem Jahr 1935 überdauerte - in seiner wesentlichen Ausgestaltung - mehr als 60 Jahre und wurde erst im Zuge der Umsetzung der EU-Binnenmarktrichtlinie 1996 mit dem Gesetz zur Neuregelung des Energiewirtschaftsrechts aus dem Jahr 1998 abgelöst. Das EnWG 1998 legte dabei nicht nur den Grundstein zur Liberalisierung der leitungsgebundenen Versorgung mit Erdgas und Elektrizität, sondern erklärte auch erstmals Umweltverträglichkeit als energiepolitische Zielsetzung im $\S 1$ des EnWG. Hiermit wurden die seit dem ersten EnWG aus dem Jahr 1935 geltenden Ziele, Versorgungssicherheit und Preisgünstigkeit, durch Hinzunahme von Umweltverträglichkeit zum heute noch gültigen energiepolitischen Zieldreieck vervollständigt. Zwei Jahre später folgte mit der Novellierung des Stromeinspeisungsgesetzes aus dem Jahr 1991 die erste Fassung des Erneuerbare-Energien-Gesetzes (EEG), welches zum Ziel hatte, ,im Interesse des Klima- und Umweltschutzes eine nachhaltige Entwicklung der Energieversorgung zu ermöglichen [...]“" (EEG 2000).

Damit wurden die wesentlichen Eckpfeiler des heutigen „Energiewenderechts“ vor knapp 20 Jahren gelegt. Seither ist das Energiewenderecht sowohl im Umfang als auch in der Komplexität erheblich gewachsen. Als einfache Indikatoren hierfür lassen sich die Anzahl der Paragraphen und die Anzahl der Wörter der jeweiligen Normen heranziehen (Waltl und Matthes 2014). Diese Entwicklung ist hier in indexierter Form dargestellt (Abb. 1). Nimmt man die Anzahl der Wörter als Maßstab, so hat sich der Umfang beider Gesetze mehr als verzwanzigfacht. Überschlägig entspricht das einer Verdopplung alle vier Jahre.

Während ein Großteil des EnWG Wachstums bis zum Jahr 2010 auf die Umsetzung der EU-Liberalisierungspakete zurückgeht, bot das EEG lange ein Innovationsumfeld mit hohen $\mathrm{Zu}$ wachsraten von Erneuerbaren Energien bei vergleichsweise geringer regulatorischer Anpassung (Böhringer et al. 2017; EWK 2014). Allerdings sind beide Vorschriften nach der Verabschiedung des sogenannten „Gesetzespaket zur Energiewende“ aus dem Jahr 2011 (Ortlieb 2011) einer besonderen Wachstumsdynamik unterworfen. Dabei ist nicht nur das Wachstum der Komplexität problematisch, sondern insbesondere auch die Regulierungsintensität und Änderungsfrequenz.
This is an article distributed under the terms of the Creative Commons Attribution License CCBY 4.0 (https://creativecommons.org/licenses/by/4.0/) https://doi.org/10.14512/tatup.28.3.48

Submitted: 28. 06. 2019. Peer reviewed. Accepted: 24.10.2019 
Angesichts der zunehmenden Diskontinuität und Dynamik des regulatorischen Rahmens herrscht unter den handelnden Akteuren große Unsicherheit. Regulatorische Risiken schweben wie ein Damoklesschwert über der Entwicklung von Geschäftsmodellen. Gesetzesänderungen können binnen kurzer Zeit - gewollt oder ungewollt - Geschäftsmodelle ermöglichen, aber genauso schnell wieder ,unwirtschaftlich regulieren“ oder streichen.

Exemplarisch ist hierfür das sogenannte „Grünstromprivileg“ zu nennen, welches eine gesetzliche Regelung im EEG 2009 zur vollständigen Zahlungsbefreiung von der EEG-Umlage für Energieversorgungsunternehmen darstellte, die - bezogen auf die gesamte von ihnen an Letztverbraucher abgegebene Strommenge mindestens $50 \%$ Strom aus erneuerbaren Energien an Letztverbraucher*innen lieferten. Über dieses Instrument zur Marktintegration wurden im Jahr 2011 mehr als $10 \%$ der gesamten EEG-Strommengen an Letztverbraucher*innen direktvermarktet (siehe Abb. 2).

Das Grünstromprivileg kreierte ein Innovationssystem aus (1) EEG-Anlagenbetreibern, (2) Marktintegratoren bzw. Energieversorgungsunternehmen und (3) Grünstromkonsument*innen. Aus Sorge um die steigernde Wirkung des Instruments auf die EEG-Umlage wurden die Regelungen im EEG 2012 verschärft und schließlich im EEG 2014 ersatzlos gestrichen. EEG-Anlagenbetreiber*innen konnten fortan ihren Strom nur noch über Direktvermarktungsunternehmen als Graustrom vermarkten, denn die ,grüne“ Eigenschaft des Stroms wurde allen Stromkunden zugeteilt. Energieversorgungsunternehmen konnten entsprechend nur noch auf Basis zumeist ausländischer Herkunftsnachweise Grünstromprodukte auflegen und vermarkten. In der Folge zerfiel die Wertschöpfungskette von EEG-Anlagenbetreiber*innen über Marktintegratoren hin zu Grünstromkonsument*innen in isolierte Fragmente. Marktakteure, die auf Basis des Grünstromprivilegs Geschäftsmodelle entwickelten, mussten diese in kürzester Zeit wieder aufgeben, umsteuern und ggf. getätigte Investitionen abschreiben.

Als ähnliche Bespielfälle lassen sich in unterschiedlicher Ausprägung die Einführung der (Teil-)EEG-Umlagepflicht auf

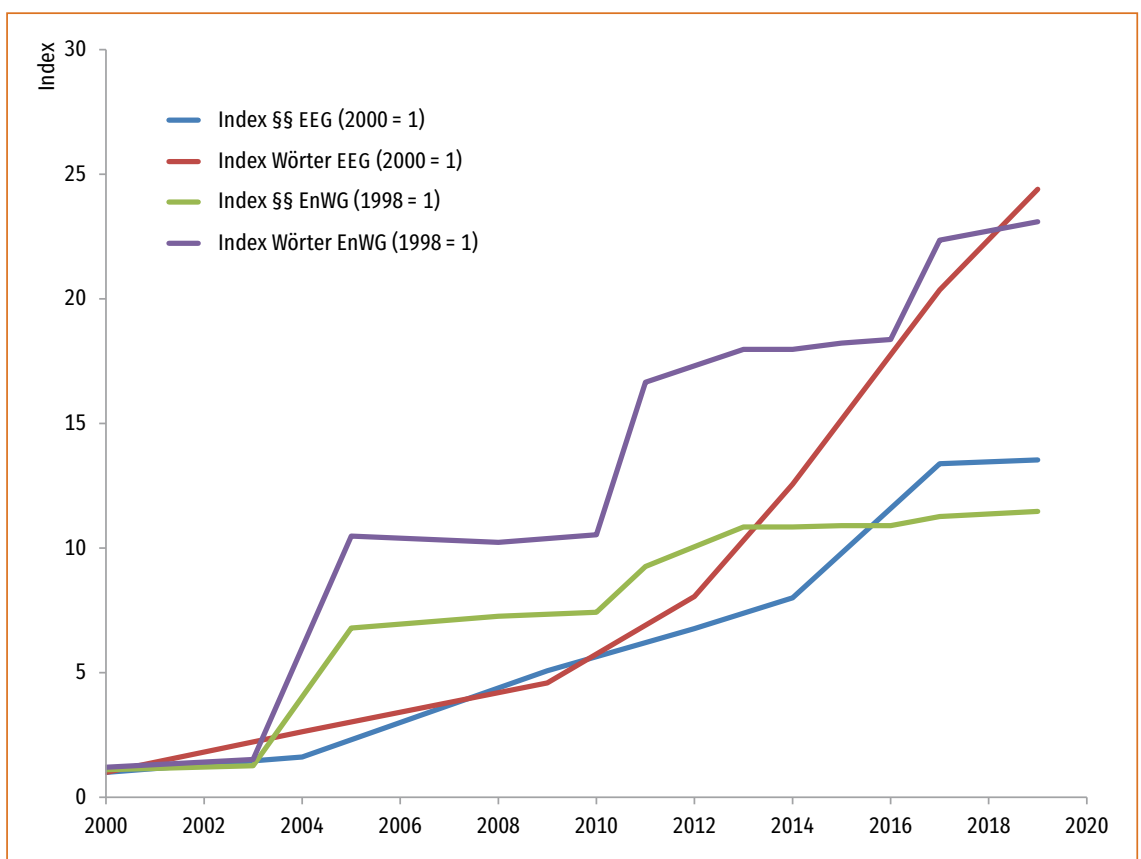

Abb.1: Entwicklung der Anzahl der Paragraphen und Wörter des EEG und des EnWG. Buchstaben-Paragraphen wurden als separate Paragraphen gezählt.

Quelle: GII 2019, Wayback Machine 2019, eigene Berechnungen

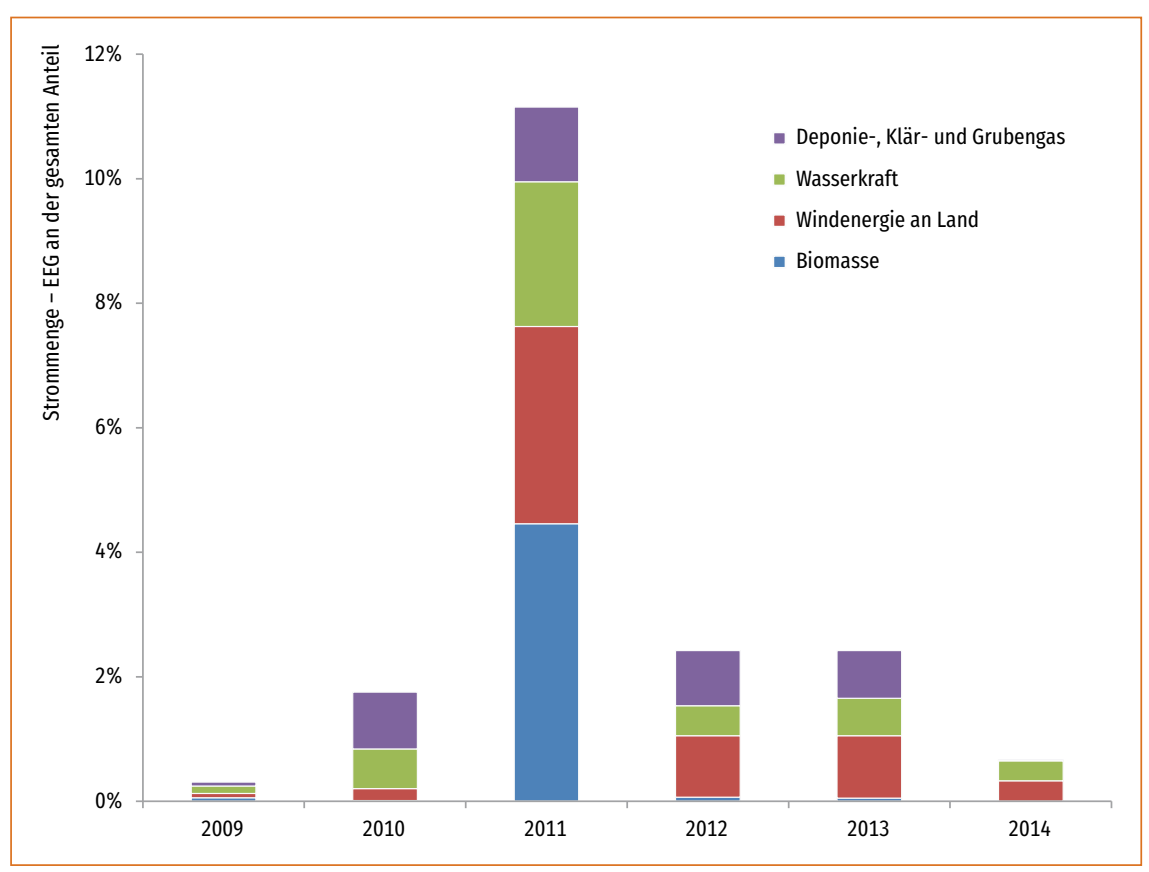

Abb. 2: Anteil der durch das Grünstromprivileg vermarkteten EEG-Strommengen.

Quelle: BMWI 2018a eigenerzeugten Eigenverbrauch im EEG 2014 oder die Änderungen zu den Regularien im Bereich des Mieterstroms nennen. Im vorliegenden Kontext geht es dabei jedoch weniger um die Frage, ob die Regularien die Richtigen waren beziehungsweise sind, sondern um die Tatsache, dass durch den Gesetzgeber in- 
stehende Rahmenbedingungen und Hemmnisse geöffnet bzw. abgebaut und Wissensdefizite reduziert werden.

Ein Beispiel hierfür ist die auf $\S 119$ Abs. 1 EnWG beruhende Verordnung zur Schaffung eines rechtlichen Rahmens zur Sammlung von Erfahrungen im Förderprogramm „Schaufenster intelligente Energie - Digitale Agenda für die Energiewende“ (SINTEG-V). Die Verordnung ist das regulatorische Grundgerüst des Förderprogramms. Sie sieht Abweichungen von bestehenden Vorschriften vor. So sollen zum Beispiel wirtschaftliche Nachteile, die aufgrund des Experiments auftreten, erstattet werden. Die am 30. Juni 2022 auslaufende Förderregel dient ausdrücklich zur Erfahrungssammlung. Die gewonnenen Erfah-

\section{Sonderwirtschaftszone}

Ein weiteres Instrument um (großflächige) Transformationsprozesse rechtlich zu gestalten sind Sonderwirtschaftszonen (SWZ). Das für die Bundesrepublik noch kaum genutzte Instrument wird auch im Rahmen des Abschlussberichts der Kommission WSB als Förderinstrument genannt (Kommission WSB 2019).

Die SWZ lässt sich rechtlich zunächst als räumlich oder sachlich begrenzter Bereich bezeichnen, in dem für bestimmte Wirtschaftsbereiche bzw. Regelungsbereiche andere Regeln gelten als in den übrigen Bereichen derselben Gerichtsbarkeit (Röhl 2004, S. 6 f.). Ausgehend vom Begriff der Wirtschaftszone und dem (vornehmlichen) Verwendungszweck dienen die SWZ der

\section{Zum Erreichen des 1,5-Grad-Ziels und damit für ein Gelingen der Energiewende bedarf es innovativer Technologien und Geschäftsmodelle.}

rungen sollen evaluiert und in einem Bericht dem Bundestag vorgelegt werden.

Obgleich Experimentierklauseln durchaus geeignet sein können Innovationen anzureizen, erweisen sie sich keineswegs als Allheilmittel. Neben der Frage der konkreten Ausgestaltung (,design matters“, Rodi 2009) bestehen für Experimentierklauseln grundsätzliche Risiken bzw. Schwächen. Als Regel-Ausnahme-Normierung stehen sie unter den Grenzen einer (strengen) Verhältnismäßigkeitsprüfung und erfordern eine hinreichende Bestimmtheit. Die Reichweite der Anwendung ist eingeschränkt und muss vom Gesetzgeber von Anfang an geregelt werden (Maßß 2001). So hat sich der Gesetzgeber auch im Rahmen der SINTEG-V für einen äußerst engen Anwendungsbereich entschieden, der systemdurchbrechende Änderungen kaum zulässt (Groebel 2019). Zudem handelt es sich bei Experimentierklauseln um Zeitgesetze, also eine befristete Regulierung. Rechtssicherheit, dass neue Technologien und Geschäftsmodelle sich nachhaltig etablieren, besteht für die Akteure damit nicht. Ein Überdauern der in der Spielwiese erprobten Neuerungen ist ungewiss.

Auch die SINTEG-V enthält ausdrücklich kein Präjudiz über zukünftige Regulierungen. Unsicherheiten und Risiken wälzt der Gesetzgeber damit auf die übrigen Akteure ab. Es lässt sich bezweifeln, ob ein Diskriminierungseingriff, der zugunsten einzelner Marktteilnehmer*innen befristete Sonderregelungen schafft, zur Entwicklung von Geschäftsmodellen und Technologie-Innnovationen führen kann (EWK 2018). Aus Sicht rational handelnder Wirtschaftssubjekte ist die Wirkung vergleichbar mit einer temporären Förderung, die nur schwerlich strategische Ressourcen zur Geschäftsmodellentwicklung oder Technologieentwicklung mobilisieren kann. Ohne weitergehende Perspektive werden wohl überwiegend lediglich Mitnahmeeffekte generiert. räumlichen Wirtschaftsförderung (Röhl 2004). Mittel dieser Förderung ist zumeist eine (größtmögliche) Deregulierung oder sonstige Form der Befreiung von Investitions- und Produktionshemmnissen (Zoll 1990).

SWZ können auch herangezogen werden, um abseits von bloßen Deregulierungen legislative und technologische Transformationsprozesse voranzutreiben bzw. zu erproben (Martinek 2018). Neben der besonderen Zugkraft für Investitionen kann die SWZ damit auch Raum für neue Entwicklungen, Technologien und Geschäftsmodelle schaffen (Yiming 2017). In Abgrenzung zu den Experimentierklauseln lassen sich SWZ räumlich als eine weitergefasste, regionale Innovationsförderung verstehen (Ginsburg 2016). Auch die Kohlekommission ordnet Sonderförderregionen den Reallaboren und Experimentierklauseln zu (Kommission WSB 2019, S. 96 f.). Sie sollen Grundlage für Modell- und Innovationsregionen sein (Kommission WSB 2019, S.97).

Wie Experimentierklauseln steht die SWZ vor der Herausforderung, zu fördernde aber bisher unbekannte Innovationen regulatorisch aufzunehmen. Im Verhältnis zur Experimentierklausel erweist sich die SWZ als nicht unwesentlich komplexer. Bereits als Förderinstrument in Form der Deregulierung erfordert die SWZ einen umfassenden Rechtsrahmen für die gesondert regulierte Region (,dual legal order“, Martinek 2018). Dabei genügt die Deregulierung keineswegs. Treten Experimentierklauseln zur Deregulierung hinzu, entsteht ein hochkomplexes (räumlich eigenständiges) Rechtsgebiet. Ob dieses im Wechselspiel mit den übrigen Regionen tatsächlich noch eine besondere Zugkraft und Innovationsförderung gewährleistet, kann zumindest bezweifelt werden. Findet die Implementierung der Innovationen ausschließlich innerhalb der SWZ statt, scheitert also die weitergehende Übertragung und Skalierung, drohen zudem systemrelevante Wirkungen auszubleiben. 


\section{$\mathrm{CO}_{2}$-Bepreisung}

Im politischen Diskurs hochaktuell und auch Gegenstand wissenschaftlicher Untersuchungen ist die $\mathrm{CO}_{2}$-Bepreisung (EWK 2016; Agora 2018; SVR 2019). Diese soll Lenkungswirkung entfalten und Innovationspotenziale heben.

$\mathrm{CO}_{2}$-Bepreisung meint ein einheitliches, staatliches Finanzierungsinstrument mit Lenkungswirkung, bei welchem der Verbrauch bzw. der Ausstoß einer gewissen $\mathrm{CO}_{2}$-Menge mit einem abzuführenden Betrag versehen wird. $\mathrm{CO}_{2}$ bekommt demnach einen Preis. Regelmäßig ist ein stetiger Anstieg des Preises vorgesehen. Durch die $\mathrm{CO}_{2}$-Bepreisung sollen treibhausgasintensive Prozesse und Produkte belastet und entsprechend treibhausgasarme Prozesse und Produkte entlastet werden.

Damit adressiert die $\mathrm{CO}_{2}$-Bepreisung nicht ausdrücklich Technologien und Geschäftsmodelle, sondern deren dekarbonisierende Wirkung. Sie ist ein technologieneutrales Instrument, welches bei einer entsprechenden Anwendung Komplexitäten abbauen kann (Agora Energiewende 2018). Mit Blick auf die regelmäßig absehbar steigende Bepreisung kann sie auch Anreize für Investitionen in innovative, treibhausgasreduzierende Technologien und Geschäftsmodelle setzen (Lenkungswirkung).

In der Schweiz besteht seit 2008 eine $\mathrm{CO}_{2}$-Abgabe auf fossile Brennstoffe. Die sogenannte Lenkungsabgabe fällt auf fossile Brennstoffe an, die in bestimmten Prozessen, bspw. der Wärme- bzw. Stromerzeugung, eingesetzt werden. Demgegenüber sind erneuerbare Brennstoffe - wie Biomasse - privilegiert. Für diese fällt die Abgabe nicht an. Der schweizerische Gesetzgeber hat für die Abgabe einen Höchstsatz von 120 Franken festgeschrieben. Die genaue Höhe der Abgabe wird dynamisch bestimmt. Sie stieg in den Jahren 2008 bis 2018 von anfangs 12 Franken, über 64 Franken, auf nunmehr 96 Franken pro Tonne $\mathrm{CO}_{2}$. Das Bundesamt für Umwelt (BAFU) attestiert der Abgabe in den einschlägigen Sektoren höhere Wirkung als anderen Instrumenten (BAFU 2018).

Abseits der Kritik an der $\mathrm{CO}_{2}$-Bepreisung wegen sozialer Härten und deren Ausgleich, kann auch die Eignung als Instrument zur energierechtlichen Zukunftsgestaltung hinterfragt werden. So kann zunächst festgehalten werden, dass nicht alle relevanten Innovationsbereiche preissensitiv sind. Ein einheitlicher Preis schafft womöglich einen fairen Wettbewerb. Werden die bestehenden Rahmenbedingungen aber im Übrigen nicht verändert, können Innovationen weiterhin an Verboten und anderen ordnungsrechtlichen Hemmnissen scheitern. Für die Wirksamkeit der $\mathrm{CO}_{2}$-Bepreisung ist die Höhe der Abgabe entscheidend. Dies zeigt auch die zum Teil drastische Erhöhung der schweizerischen $\mathrm{CO}_{2}$-Abgabe, die sonst weitestgehend wirkungslos geblieben wäre. Auch die $\mathrm{CO}_{2}$-Bepreisung ist ein letztlich zeitlich beschränktes Instrument. Mit stetigem Nähern des Ziels einer klimaneutralen Gesellschaft, sinkt auch die Lenkungswirkung der $\mathrm{CO}_{2}$-Bepreisung. Darüber hinaus adressiert eine $\mathrm{CO}_{2}-\mathrm{Be}-$ preisung lediglich einen - wenn auch wesentlichen - Treiber des Klimawandels. Das Energiewenderecht ist jedoch kein bloBes Emissionseinsparungsrecht. Vielmehr zielt es insgesamt auf einen erhöhten Umweltschutz, sodass auch andere Bereiche der
Energiewende und Innovationen berücksichtigt werden müssen. $\mathrm{Zu}$ diesen gehören bspw. Fragen der Biodiversität und des Flächenverbrauchs.

\section{Fazit}

Mit Blick auf den Transformationsprozess der Energiewende und die hierfür notwendigen Innovationen bleiben erhebliche Herausforderungen, technologisch, ökonomisch, gesellschaftlich und auch rechtlich. Zur Bewältigung dieser Herausforderungen steht dem Gesetzgeber eine große Instrumentenauswahl zur Verfügung. Die Untersuchung hat gezeigt, dass die derzeit debattierten Instrumente für sich allein kein Garant für Innovation und Erfolg der Energiewende sind. Ausgehend von einer langfristigen, robusten Strategie zum Senken der Treibhausgasemissionen - etwa durch eine stetig ansteigende $\mathrm{CO}_{2}$-Bepreisung - kann der Gesetzgeber die energierechtliche Zukunftsgestaltung erfolgversprechend angehen. Diese Strategie kann und muss durch einzelne Experimentierklauseln oder SWZ unterstützt und mitgetragen werden. Dem folgend steht dem Gesetzgeber ein geeignetes Handwerkszeug zur Verfügung, mit dem er den Weg des Transformations- und Innovationsprozesses erfolgreich gestalten kann.

\section{Literatur}

AGEE-Stat - Arbeitsgruppe Erneuerbare Energien-Statistik (2019): Zeitreihen zur Entwicklung der erneuerbaren Energien in Deutschland (Stand: Februar 2019) Online verfügbar unter https://www.erneuerbare-energien.de/EE/Redaktion/ DE/Downloads/zeitreihen-zur-entwicklung-der-erneuerbaren-energien-indeutschland-1990-2018-excel.xlsx, zuletzt geprüft am 11.04.2019.

Agora Energiewende (2018): Eine Neuordnung der Abgaben und Umlagen auf Strom, Wärme, Verkehr. Optionen für eine aufkommensneutrale $\mathrm{CO}_{2}$-Bepreisung von Energieerzeugung und Energieverbrauch. Online verfügbar unter https://www.agora-energiewende.de/fileadmin2/Projekte/2017/Abgaben_ Umlagen/147_Reformvorschlag_Umlagen-Steuern_WEB.pdf, zuletzt geprüft am 18.06.2019.

BAFU - Bundesamt für Umwelt (2018): Faktenblatt Wirkungsabschätzung und Evaluation der C02-Abgabe auf Brennstoffe. Online verfügbar unter https:// www.bafu.admin.ch/dam/bafu/de/dokumente/klima/fachinfo-daten/ faktenblatt_1_c02-abgabe_auf_brennstoffe.pdf.download.pdf/faktenblatt_1_ c02-abgabe_auf_brennstoffe.pdf, zuletzt geprüft am 17.10.2019.

BMU - Bundesministerium für Umwelt, Naturschutz und nukleare Sicherheit (2019): Klimaschutzplan 2050. Klimaschutzpolitische Grundsätze und Ziele der Bundesregierung. Online verfügbar unter https://www.bmu.de/ fileadmin/Daten_BMU/Download_PDF/Klimaschutz/klimaschutzplan_2050_ bf.pdf, zuletzt geprüft am 27.06.2019.

BMWi - Bundesministerium für Wirtschaft und Energie (2018a): EEG in Zahlen. Vergütungen, Differenzkosten und EEG-Umlage 2000 bis 2019 (Stand: 15. Oktober 2018). Online verfügbar unter https://www.erneuerbareenergien.de/EE/Redaktion/DE/Downloads/eeg-in-zahlen-pdf.pdf\%3F blob\%3DpublicationFile, zuletzt geprüft am 17.10.2019.

BMWi (2018b): Reallabore. Testräume für Innovation und Regulierung. Online verfügbar unter https://www.bmwi.de/Redaktion/DE/Dossier/reallabore- 
testraeume-fuer-innovation-und-regulierung.html, zuletzt geprüft am 18.06.2019.

BNetzA - Bundesnetzagentur (2019): Kraftwerksliste Bundesnetzagentur. Stand: 07. März 2019. Bundesnetzagentur für Elektrizität, Gas, Telekommunikation, Post und Eisenbahnen. Bonn. Online verfügbar unter https://www.bundesnetzagentur.de/DE/Sachgebiete/ElektrizitaetundGas/ Unternehmen_Institutionen/Versorgungssicherheit/Erzeugungskapazitaeten/ Kraftwerksliste/kraftwerksliste-node.html, zuletzt geprüft am 19.05.2019.

Böhringer, Christoph; Cuntz, Alexander; Harhoff, Dietmar; Asane-Otoo, Emmanuel (2017): The impact of the German feed-in tariff scheme on innovation. Evidence based on patent filings in renewable energy technologies. In: Energy Economics 67 (C), S. 545-553.

Diehl, Andrea (2014): Innovationsfördernde dynamische Regulierung. BadenBaden: Nomos.

Ekardt, Felix; Wieding, Jutta; Zorn, Anika (2018): Paris agreement, precautionary principle and human rights. Zero emissions in two decades? Online verfügbar unter https://www.mdpi.com/2071-1050/10/8/2812, zuletzt geprüft am 12.09.2019.

EWK - Expertenkommission zum Monitoring-Prozess „Energie der Zukunft“ (2012): Stellungnahme zum ersten Monitoring-Bericht der Bundesregierung für das Berichtsjahr 2011. Berlin: o. V.

EWK (2014): Stellungnahme zum ersten Fortschrittsbericht der Bundesregierung für das Berichtsjahr 2013. Berlin: 0. V.

EWK (2016): Stellungnahme zum fünften Monitoring-Bericht der Bundesregierung für das Berichtsjahr 2015. Berlin: 0.V.

EWK (2018): Stellungnahme zum fünften Monitoring-Bericht der Bundesregierung für das Berichtsjahr 2017. Berlin: o. V.

Franzius, Claudio (2015): Regulierung und Innovation im Mehrebenensystem. In: Die Verwaltung 48, S. 175-201.

Gawel, Erik (2009): Technologieförderung durch „Stand der Technik“. In: Martin Eifert und Wolfgang Hoffmann-Riem (Hg.): Innovationsfördernde Regulierung. Berlin: Duncker \& Humblot, S. 197-220.

GII (2019): „Gesetze im Internet“. Online verfügbar unter www.gesetze-iminternet.de, zuletzt geprüft am 18. 04.2019.

Ginsburg, Tom (2016): Special economic zones. A constitutional political economy perspective. In: Jürgen Basedow und Toshiyuki Kono (Hg.): Special economic zones. Law and policy perspectives. Tübingen: Mohr Siebeck, S. 130-141.

Groebel, Annegret (2019): §119a EnWG. In: Jürgen Säcker (Hg.): Berliner Kommentar zum Energierecht. Frankfurt am Main: dfv Mediengruppe, S. 4037-4046.

Kommission WSB - Kommission „Wachstum, Strukturwandel und Beschäftigung“ (2019): Abschlussbericht. Online verfügbar unter https://www.bmwi.de/ Redaktion/DE/Downloads/A/abschlussbericht-kommission-wachstumstrukturwandel-und-beschaeftigung.pdf?__blob=publicationFile\&v=4, zuletzt geprüft am 18. 06.2019.

Korff, Rüdiger (2016): Resilienz. Eine Frage von Biegen oder Brechen im Ausnahmefall. In: Kai von Lewinski (Hg.): Resilienz des Rechts. Baden-Baden: Nomos, S. 23-32.

Martinek, Madeleine (2018): Experimental legislation in China between efficiency and legality. Cham: Springer.

Maaß, Volker (2001): Experimentierklauseln für die Verwaltung und ihre verfassungsrechtlichen Grenzen. Berlin: Duncker \& Humblot.

Ortlieb, Birgit (2011): Das Gesetzespaket zur Energiewende. Zusammenfassender Überblick über die wesentlichen Inhalte der acht am 8. Juli 2011 endgültig verabschiedeten, mittlerweile im Bundesgesetzblatt veröffentlichten (und zum größten Teil auch bereits in Kraft getretenen) Gesetze. In: EWeRK 11 (4), S. 151-156.

Rodi, Michael (2009): Innovationsförderung durch ökonomische Instrumente der Umweltpolitik. In: Martin Eifert und Wolfgang Hoffmann-Riem (Hg.): Innovationsfördernde Regulierung. Berlin: Duncker \& Humblot, S. 146-169.

Röhl, Klaus-Heiner (2004): Sonderwirtschaftszonen als Instrument der Regionalentwicklung. Neue Ideen für die neuen Bundesländer. Köln: Deutscher Instituts-Verlag.

Roßnagel, Alexander (2007): Innovation als Gegenstand der Rechtswissenschaft. In: Hagen Hof und Ulrich Wengenroth (Hg.): Innovationsforschung - Ansätze, Methoden, Grenzen und Perspektiven. Münster: Lit, S. 9-22.

SVR - Sachverständigenrat zur Begutachtung der gesamtwirtschaftlichen Lage (2019): Aufbruch zu einer neuen Klimapolitik. Sondergutachten. Online verfügbar unter https://www.sachverstaendigenrat-wirtschaft.de/fileadmin/ dateiablage/gutachten/sg2019/sg_2019.pdf, zuletzt geprüft am 25. 09.2019.

Waltl, Bernhard; Matthes, Florian (2014): Towards measures of complexity. Applying structural and linguistic metrics to German laws. $27^{\text {th }}$ International Conference on Legal Knowledge and Information Sytems. Krakau, Polen, 10. 12.2014.

Wayback Machine (2019): Internet archive. Online verfügbar unter http://web. archive.org/, zuletzt geprüft am 25.04.2019.

Yiming, Yuan (2017): The dynamic evolution of China's special economic zones and their practice. In: Yuan Yiming (Hg.): Studies on China's special economic zones. Singapur: Springer, S. 13-22.

Zoll, Jürgen (1990): Freizonen im internationalen Wirtschaftsrecht. Völkerrechtliche Schranken exzessiver Wirtschaftsförderung. Baden-Baden: Nomos.

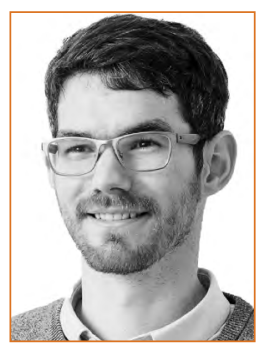

MICHAEL KALIS

forscht seit 2018 am IKEM zu den rechtlichen Rahmenbedingungen von Experimentierklauseln, Reallaboren und grünen Gasen.

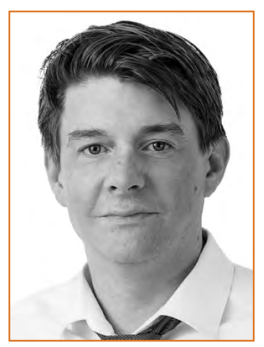

\section{LARS DITTMAR}

erforscht seit 2019 am IKEM wirtschaftswissenschaftliche und technische Fragenstellungen der Energiewende. Zuvor unterstütze er seit der Gründung im Jahr 2011 die Expertenkommission des Energiewende-Monitorings in Deutschland und analysierte in diesem Zusammenhang die Fortschritte der Energiewende. 